\title{
Asymmetrical Attention to Gambling-related Stimuli and Pupillary Dilation in Response to Wins and Losses in Problem Gamblers
}

Yayoi Shigemune ( $\sim$ gemune@gmail.com )

Chuo University

\section{Akira Midorikawa}

Chuo University

\section{Research Article}

Keywords: Gambling disorder, problem gambling, eye tracking, gaze allocation, pupillary diameter

Posted Date: November 30th, 2021

DOl: https://doi.org/10.21203/rs.3.rs-1080574/v1

License: (a) (1) This work is licensed under a Creative Commons Attribution 4.0 International License.

Read Full License 


\section{Abstract}

Gambling disorder and problem gambling are characterized by persistent and repetitive problematic gambling behavior. Attentional bias toward gambling-related stimuli such as casino chips, dice, roulette, etc. have been observed in problem gamblers (PGs), but it remains unclear whether stimuli in gambling tasks elicit greater attention and pupillary responses in PGs. To address this issue, we administrated PGs and non-problem gamblers (NPGs) a gambling task accompanied by eye-tracking measurements, in which the participants were required to choose one of the paired pictures to receive monetary rewards and avoid punishments. Concerning attentional allocation, PG showed a greater attentional preference for the right-hand pictures in the decision and feedback phases, and compared to NPGs, PGs' attention was narrower and more focused on the left-hand pictures in the decision phase. Concerning pupillary dynamics indicative of noradrenergic locus coeruleus activity, pupillary dilation in response to rewards and punishments was observed only in PGs. Studies of unilateral spatial neglect have proposed that asymmetric attention is induced by hemispheric imbalance. Accordingly, asymmetrical allocation of attention by PGs may reflect hemispheric imbalance, and pupillary dynamics may reflect sensitivity to wins and losses.

\section{Introduction}

Gambling disorder, previously known as pathological gambling, is defined as persistent and recurrent problematic gambling behavior leading to clinically significant impairment or distress. ${ }^{1}$ Problem gambling can lead to financial and social difficulties, psychiatric comorbidities, poor physical health, suicide, and criminal behaviors. ${ }^{2-4} \mathrm{~A}$ recent public health review suggested that the increasing availability of, and participation in, online gambling has increased the prevalence of gambling disorder and contributed to associated morbidities and other gambling-related problems. ${ }^{5}$ To address these growing problems, there is a need to elucidate the pathology of gambling disorder and problem gambling. An eye tracker is an effective device for measurement of physiological responses, such as gaze allocation and pupillary diameter. Gaze allocation assessment enables identification of salient objects, while pupillary diameter assessment can be used to determine the salience of objects. Thus, simultaneous gaze allocation and pupillary diameter assessment can help examine cognitive processing in problem gamblers (PGs) from two aspects. In this study, we used an eye tracker to investigate differences in attentional allocation and pupillary dynamics between PGs and non-problem gamblers (NPGs) during a gambling task.

Previous studies concerning attentional allocation during gambling tasks have indicated that participants gazed at the stimuli they ultimately selected before selecting them, ${ }^{6}$ and that stress and sensory cues denoting a win modulated attentional allocation and promoted riskier choices. ${ }^{7,8}$ However, attentional allocation in PGs during gambling tasks has not been considered. A systematic review found that PGs and pathological gamblers exhibited an attentional bias toward gambling-related stimuli, such as the words casino, poker, and jackpot, and toward pictures of poker chips, roulette, and dice, compared to 
neutral stimuli. ${ }^{9}$ Brevers et al. reported that PGs could detect changes in gambling-related stimuli more rapidly, and that the stimuli were more likely to elicit the first saccade and a longer fixation. Therefore, we hypothesized that PGs would show greater attention, even to neutral stimuli, in a gambling task compared to NPGs.

Pupillary diameter is modulated by the noradrenergic locus coeruleus and reflects various cognitive processes and emotions, such as attraction, mental effort, surprise, attention, and exploration. ${ }^{10,11}$ Previous studies using non-gambling tasks have demonstrated that greater pupillary dilation occurs during reward anticipation, ${ }^{12,13}$ and that excessive effects of reward magnitude on pupillary diameter were predictive of subsequent failure in a stopwatch task. ${ }^{14}$ Pupillary diameter has been presumed to be modulated by uncertainty and violations of expectations during gambling. ${ }^{15-17}$ However, pupillary dynamics in PGs during gambling tasks remain unexamined. We hypothesized that pupillary dynamics would differ between PGs and NPGs in gambling tasks due to differences in reward and punishment predictions.

In this study, PGs and NPGs were chosen from among 100 gamblers on the basis of their South Oaks Gambling Screen (SOGS) scores. ${ }^{18}$ The gambling task was composed of decision, feedback, and fixation phases, with eye-tracking (Fig. 1). In the decision phase, pairs of pictures were presented on the left- and right-hand sides of the screen; PGs and NPGs were required to choose one of the two pictures. In the feedback phase, the PGs and NPGs received a monetary reward or a punishment if the selected picture was framed by a red (reward condition) or blue line (punishment condition). If the chosen picture was framed by a gray line (control condition) or the unchosen picture was framed by red, blue, or gray lines, there was no reward or punishment. Half of the chosen pictures were framed (congruent condition) while the other half were not (incongruent condition), in each of the reward, punishment, and control conditions. This enabled comparison between PGs and NPGs in terms of attentional allocation to stimuli in a gambling task and the modulation of pupillary dynamics by wins and losses.

(Figure 1 near here)

\section{Results}

\section{Experimental gambling task}

The results of the gambling task were analyzed to confirm response bias to the left- or right-hand pictures (Table 1). Two-sample t-tests showed no statistically significant differences in the left/right response indices between the PGs and NPGs $[t(42)=0.81, p=0.42, r=0.12]$. Two-way analysis of variance (ANOVA) of the response times, with side and group as the independent variables, revealed no statistically significant main or interaction effects [main effect of side: $F(1,42)=1.14, p=0.29, n p 2=$ 0.03; main effect of group: $F(1,42)=2.31, p=0.14, \eta p 2=0.05$; interaction between side and group: $F(1,42)=0.01, p=0.90, \eta p 2=0.00]$. Thus, no response biases were identified during the task. 
Table 1

Results of experimental task and test battery (mean $\pm S D$ ).

\begin{tabular}{|c|c|}
\hline $\begin{array}{l}\text { Problem gamblers } \\
\text { (PGs) }\end{array}$ & $\begin{array}{l}\text { Non-problem gamblers } \\
\text { (NPGs) }\end{array}$ \\
\hline
\end{tabular}

\section{Experimental task}
Left/right index
$-1.4 \pm 21.2$
$-6.0 \pm 15.7$
$0.81 \quad 0.42$
0.12

\section{Digit span}

$\begin{array}{llllll}\text { Forward } & 8.9 \pm 1.8 & 9.0 \pm 1.7 & -0.09 & 0.93 & 0.01 \\ \text { Backward } & 7.3 \pm 1.7 & 7.3 \pm 2.4 & -0.07 & 0.94 & 0.01\end{array}$

\section{Rey-Osterrieth Complex Figure Test}

$\begin{array}{llllll}\text { Copy } & 35.1 \pm 1.0 & 35.4 \pm 0.9 & -1.25 & 0.22 & 0.19 \\ \text { Retrieval } & 23.7 \pm 6.2 & 25.5 \pm 5.0 & -1.03 & 0.31 & 0.16\end{array}$

Behavioral Inhibition System/Behavioral Approach System

\begin{tabular}{lccccc|}
$\begin{array}{l}\text { Behavioral Inhibition } \\
\text { System }\end{array}$ & $17.8 \pm 3.3$ & $18.0 \pm 3.4$ & -0.22 & 0.82 & 0.03 \\
\hline $\begin{array}{l}\text { Behavioral Approach } \\
\text { System }\end{array}$ & $43.9 \pm 4.2$ & $37.5 \pm 5.3$ & 4.5 & $0.00 * *$ & 0.57 \\
\hline
\end{tabular}

\section{Sensitivity to Punishment and Sensitivity to Reward Questionnaire}

$\begin{array}{llllll}\text { Sensitivity to } & 63.5 \pm 12.1 & 61.5 \pm 8.8 & 0.61 & 0.54 & 0.1\end{array}$

Punishment

$64.5 \pm 7.9$

$49.8 \pm 7.3$

$6.40 .00^{\star *}$

0.7

\section{Sensation Seeking Scale - Abstract Expression}

\begin{tabular}{lccccc}
$\begin{array}{l}\text { Thrill and Adventure } \\
\text { Seeking }\end{array}$ & $16.1 \pm 4.4$ & $10.2 \pm 3.4$ & 5.03 & $0.00^{\star *}$ & 0.61 \\
\hline $\begin{array}{l}\text { Disinhibition } \\
\text { Experience Seeking }\end{array}$ & $16.9 \pm 3.9$ & $12.6 \pm 3.8$ & 3.73 & $0.00^{\star \star}$ & 0.5 \\
\hline SD $=$ standard deviation & $22.2 \pm 3.1$ & $16.8 \pm 4.5$ & 4.69 & $0.00^{\star *}$ & 0.59 \\
$* *=p<0.01, *=p<0.05,+=p<0.1$, & & & &
\end{tabular}

\section{Test battery}

A test battery, including the forward and backward digit span tasks, ${ }^{19}$ Rey-Osterrieth Complex Figure test, ${ }^{20,21}$ BIS/BAS scales, ${ }^{22}$ Sensitivity to Punishment and Sensitivity to Reward Questionnaire (SPSRQ), ${ }^{23}$ and Sensation Seeking Scale-Abstract Expression (SSS-AE), ${ }^{24,25}$ were administered to test for group 
differences in attention, visuospatial cognition, episodic memory, and personality traits. Two-sample ttests showed significantly higher scores for PGs than NPGs on the BAS [t(42) $=4.50, p<0.01, r=0.57$, sensitivity to reward $(S R)[t(42)=6.40, p<0.01, r=0.70]$, and multiple $S S S-A E$ subscales [thrill and adventure seeking ( $t[42]=5.03, p<0.01, r=0.61)$, disinhibition $(t[42]=3.73, p<0.01, r=0.50)$, and experience seeking $(\mathrm{t}[42]=4.69, \mathrm{p}<0.01, \mathrm{r}=0.59)]$. There were no group differences in any other scores (Table 1).

\section{Gaze allocation}

The proportions of validated measurements were sufficiently high and did not differ between the PGs (mean: 90.2, standard deviation [SD]: 8.8) and NPGs [mean: 91.0, SD: 7.1; t(42) $=-0.32, p=0.75, r=0.05$ ]. For each pixel, the ratios of gaze-allocation times in the decision, feedback, and fixation phases were calculated for both PGs and NPGs (Fig. 2a). Their gaze was concentrated on the pictures in the decision and feedback phases, and on the fixation cross in the fixation phase, in both the PGs and NPGs. Pixels with significant ratio differences [PGs > NPGs (PG-dominant pixels) or NPGs > PGs (PG-dominant pixels)] were identified by two-sample t-tests in the decision, feedback, and fixation phases (Fig. 2b). The PGs' and NPGs' peak ratios were similar, and the PG-dominant pixels were concentrated around the peaks. In the peripheral parts, the PG-dominant pixels were intermixed with NPG-dominant pixels. The midpoints between peaks for the PGs and NPGs were defined as the foci, and the PG- and NPG-dominant pixels were counted at intervals of 10 pixels from the foci (Fig. 2c). Around all foci, the proportion of PGdominant pixels was greater than that of NPG-dominant pixels. In peripheral areas, the reverse situation (higher proportion of NPG- than PG-dominant pixels) was observed only on the left side in the decision phase. In both the decision and feedback phases, the proportion of PG-dominant pixels around the foci was greater on the right side than on the left side. Thus, two-way ANOVAs were performed on the sum of the gaze allocation times for the pixels corresponding to the left and right pictures. These analyses showed a significant interaction between side (left and right) and group (PGs and NPGs) in the decision and feedback phases [decision phase: $F(1,42)=6.46, p<0.05, \eta p 2=0.13$; feedback phase: $F(1,42)=6.06$, $p<0.05, \eta p 2=0.13$ ] (Fig. 3). Post hoc analyses revealed that the ratio of gaze allocation times was greater on the right side than on the left side in PGs $(p<0.05)$, and that the ratio of gaze allocation on the right side was greater in PGs than NPGs $(p<0.05)$. The main effect of side was statistically significant (right $>$ left) in the feedback phase $[F(1,42)=4.34, p<0.05, n p 2=0.09]$. No other main or interaction effects were statistically significant in the decision, feedback, or fixation phases ( $p>0.1$ for all comparisons).

\section{Pupillary dynamics}

The temporal dynamics of pupillary diameter were analyzed in each experimental condition based on the factors of motivation (reward, punishment, and control) and result (congruent and incongruent) (Fig. 4a). To identify changes in pupillary diameter related to receiving rewards and punishments, differences in zvalues between congruent and incongruent conditions were calculated and two-sample t-tests were used to compare them between the reward and control conditions, and between the punishment and control conditions (Fig. 4b). In the PGs, significant reward-related differences were observed in the fixation phase, 
while punishment-related significant differences were observed in the decision, feedback, and fixation phases. These differences were not observed in the NPGs.

\section{Discussion}

In this study, eye-tracking measurements were performed during a gambling task to compare gaze allocation and pupillary dynamics between PGs and NPGs. Gaze allocation was generally more concentrated around the peaks in PGs than in NPGs. In addition, more narrowly focused gaze was observed for the left-hand pictures in the decision phase in NPGs than in PGs. Furthermore, in the decision and feedback phases, gaze allocation was greater to the right picture than to the left picture in PGs, but not in NPGs. Only PGs showed significant differences in pupillary dynamics between the reward and control conditions, and between the punishment and control conditions. To our knowledge, this study is the first to demonstrate distinctive gaze allocation and pupillary dynamics characteristics in PGs while engaged in gambling.

Consistent with our hypothesis regarding attentional allocation, higher ratios of gaze allocation time were observed in PGs than in NPGs around the peaks in the decision, feedback, and fixation phases. Previous studies indicated that PGs had attentional bias to gambling-related stimuli compared to neutral stimuli. 9,26 Unlike previous studies that used non-gambling tasks (e.g., Stroop, flicker, and lexical salience tasks) (Brevers et al. 2011; McCusker and Gettings 1997; Zack and Poulos 2004), the PGs received rewards and avoided punishments based on their choice of neutral stimuli in this study. They may, therefore, have concentrated not only on the stimuli but also on the overall task. This would be consistent with the finding that their attentional allocation was greater even in the fixation phase. While PGs gazed at the left-hand pictures more than the NPGs during the decision phase, peripheral gaze was "higher" for NPGs than PGs. This was presumably because PGs inspected the pictures more carefully, in an attempt to identify winning strategies that did not actually exist, while the NPGs explored a wider range of pictures. However, this difference was not observed for the right-hand pictures, possibly due to the masking effect of an attentional preference for the right-hand pictures in PGs. Left unilateral spatial neglect due to right hemispheric injury has been reported in neuropsychological studies. ${ }^{30}$ Heilman et al. proposed a theoretical model of unilateral spatial neglect, in which the left hemisphere directs attention to the right side, and the right hemisphere to both sides. ${ }^{31}$ Based on this model, increased activity in the left hemisphere or decreased activity in the right hemisphere may result in an attentional bias to the right side in PGs. Davidson et al. proposed a model of cerebral asymmetry and approach-withdrawal, in which the left hemisphere exhibited greater activation during approach and the right hemisphere exhibited greater activation during withdrawal. This model has been supported by numerous studies. ${ }^{32-34}$ Some studies have indicated that this hemispheric asymmetry influences BIS/BAS scores, ${ }^{35-37}$ whereby activation of the left hemisphere was greater in participants with higher BAS scores and they made more disadvantageous choices in the lowa Gambling Task. ${ }^{38,39}$ In the present study, PGs had significantly higher BAS, SR, and SSS-AE thrill and adventure seeking and experience seeking subscale scores. In another study, higher novelty-seeking scores were associated with greater rightward attentional bias, and 
it was suggested that this bias was related to dopamine asymmetry favoring the left hemisphere. ${ }^{40}$ Thus, excessive activity in the left hemisphere may lead to an attentional preference to the right side in PGs.

Consistent with our hypothesis regarding pupillary dynamics, the differences in z-values between the congruent and incongruent conditions reflected significant differences between the reward and control conditions, and between the punishment and control conditions, only in PGs. Because pupillary dilation reflects activity in noradrenergic locus coeruleus neurons, ${ }^{10,11}$ the sympathetic nervous system in PGs may be excited by rewards and punishments. Previous studies reported pupillary dilation during reward anticipation and violation of expectation. ${ }^{12-17}$ Significant differences in responses to punishment were observed mainly in the feedback phase, while differences in responses to reward were observed in the fixation phase. Therefore, punishments may surprise PGs, and rewards may enhance reward anticipation for the next trial. However, significant differences in responses to punishment were also observed in the decision phase, although there were no discernible differences between the conditions. Previous studies have reported irrational behavior during gambling (i.e., chasing losses), whereby people make riskier decisions after a loss than after a win, and decide to continue gambling despite considerable losses (Brevers et al. 2017; Campbell-Meiklejohn et al. 2008; Gainsbury et al. 2014). It is possible that prior trials may influence the neural activity of PGs in the decision phase.

Our investigations of attentional allocation and pupillary dynamics during gambling tasks indicate that there are two distinctive cognitive processing pathways in PGs. First, PGs allocate more attention to the right side because of asymmetrical activation favoring the left hemisphere. Second, PGs show pupillary dilation in association with noradrenergic locus coeruleus activity because of punishment-mediated surprise and expectations of future rewards. These results also demonstrate the advantages of samplespecific analysis in terms of both attentional allocation and pupillary dynamics to avoid canceling out significant differences by data integration. In fact, the significantly greater attentional allocation to the left-hand pictures by PGs in the sample-specific analysis was not observed in the analysis of gaze allocation toward the whole picture. However, problematic behaviors in PGs could not be identified because the outcomes of gambling were completely controlled. Future studies should examine how these differences in attentional allocation and pupillary dynamics are related to cognitive abnormalities and behaviors in problem gambling and gambling disorder.

\section{Methods}

\section{Participants}

One hundred individuals (mean \pm SD age: $39.2 \pm 11.2$ years; 49 men and 51 women) who had previously engaged in gambling were assessed using the SOGS and Massachusetts Gambling Screen ${ }^{18,44}$. SOGS scores $\geq 5$ were regarded as indicative of probable pathological gambling, and scores of 3-4 were regarded as possible pathological gambling. There were 21 probable gamblers and 1 possible gambler among the PGs (mean \pm SD age: $38.5 \pm 11.2$ years; 15 men and 7 women). After matching for age, years of education, and sex, 22 participants with SOGS scores of $0-1$ were included in the NPG group (mean \pm 
SD age: $38.7 \pm 10.4$ years; 13 men and 9 women) (Table 2 ). The optimal sample size was determined based on a G-Power analysis, ${ }^{45}$ for a power of 0.95 , medium effect size of $F=0.25$ for the ANOVA, and a level of 0.05. The SOGS and Massachusetts Gambling Screen scores showed significant differences between the PGs and NPGs $[t(22.6)=16.88, p<0.001, r=0.96 ; t(21.3)=7.17, p<0.001, r=0.84$, respectively]. The protocol was approved by the ethics committee of Chuo University. All participants provided written informed consent in accordance with the Declaration of Helsinki.

Table 2

Demographic data.

\begin{tabular}{|llllll|}
\hline & $\begin{array}{l}\text { Problem } \\
\text { gamblers (PGs) }\end{array}$ & $\begin{array}{l}\text { Non-problem } \\
\text { gamblers (NPG) }\end{array}$ & t/ $\chi^{2}$ & p & r/V \\
\hline Age, years (mean \pm SD) & $38.5 \pm 11.2$ & $38.7 \pm 10.4$ & -0.04 & 0.97 & 0.01 \\
\hline Age range, years & $22-57$ & $22-57$ & & & \\
\hline Sex (male: female) & $15: 07$ & $13: 09$ & 0.39 & 0.53 & 0.09 \\
\hline Years of education (mean \pm SD) & $15.2 \pm 1.6$ & $15.5 \pm 1.1$ & -0.86 & $0.40 * *$ & 0.13 \\
\hline $\begin{array}{l}\text { South Oaks Gambling Screen } \\
\text { score (mean } \pm \text { SD) }\end{array}$ & $8.2 \pm 2.2$ & $0.2 \pm 0.4$ & 16.88 & $0.00 * *$ & 0.96 \\
\hline $\begin{array}{l}\text { Massachusetts Gambling Screen } \\
\text { score (mean } \pm \text { SD) }\end{array}$ & $4.0 \pm 2.6$ & $0.0 \pm 0.2$ & 7.17 & $0.00 * *$ & 0.84 \\
\hline SD $=$ standard deviation & & & & & \\
\hline$* *=p<0.01, *=p<0.05,+=p<0.1$ & & & & & \\
\hline
\end{tabular}

\section{Stimuli}

In total, 120 pairs of pictures of non-living objects (e.g., daily goods, furniture, vehicles, and clothes) were chosen from a dataset used in previous studies ${ }^{46}$. In the experimental task, some pictures were presented on the left-hand side of the computer screen, and others were presented on the right-hand side (Fig. 1). The pairs of pictures were divided into six homogeneous sets, with similar levels of complexity, familiarity, arousal, and valence between the left and right sides ( $p \geq 0.1$ in all comparisons). Furthermore, the level of similarity between pairs of pictures did not differ among the sets $(p \geq 0.1)$. The combinations of stimulus sets and experimental conditions were counterbalanced.

\section{Experimental task}

Gaze positions and pupillary diameters were obtained from both eyes using a Tobii Pro Nano eye tracker and Tobii Pro Lab Presenter version 1.152 (Tobii Technology, Stockholm, Sweden). The sampling rate was set at $60 \mathrm{~Hz}$, and the screen resolution was set to 1,024 × 768 pixels. To calibrate the eye gaze position on the screen, a standard nine-point calibration procedure was conducted before the experimental task. The experimental task consisted of two 60 -trial sessions and was conducted using 
MATLAB R2010b (MathWorks, Natick, MA, USA) and Cogent 2000 version 1.33 (www.vislab.ucl.ac.uk/cogent.php). Each trial was composed of a 3-second decision phase, 1.5-second feedback phase, and 1-second fixation phase. In the decision phase, the pairs of pictures and a fixation cross were presented, and participants were required to choose one of the pictures. A yellow inverted triangle was presented above the chosen picture after it had been selected. In the feedback phase, one of the pictures was framed with a red (reward condition), blue (punishment condition), or gray (control condition) line. If the chosen picture was framed by a red line, the participant won 100 yen (equivalent to approximately 1 US dollar). If the chosen picture was framed by a blue line, the participant lost 100 yen. If the chosen picture was framed by a gray line, or if the unchosen picture was framed by a red, blue, or gray line, the participant would neither win nor lose any money. In the fixation phase, only the fixation cross was shown on the screen. Participants were instructed to maximize gain and minimize loss. In each of the reward, punishment, and control conditions, half of the chosen pictures were framed (congruent condition) and the remaining half were not framed (incongruent condition).

\section{Test battery}

After finishing the experimental task, the participants completed a test battery that included the forward and backward digit span tasks, ${ }^{19}$ Rey-Osterrieth Complex Figure test,${ }^{20,21}$ BIS/BAS scales, ${ }^{22}$ SPSRQ, ${ }^{23}$ and SSS-AE. 24,25

\section{Data analysis of behavioral data}

The left/right index was defined as [(right decisions - total decisions/2)/(total decisions/2)] $\times 100$. A left/right index of -100 indicated that the participant always chose left, and a left/right index of 100 indicated that the participant always chose right. To determine whether responses differed between PGs and NPGs, the left/right indices were analyzed by two-sample t-tests. The response times were also analyzed by two-way ANOVA, with side (left and right) and group (PGs and NPGs) as the independent variables. Two-sample t-tests between PGs and NPGs were performed for each component of the test battery. IBM SPSS Statistics was used for statistical analyses (version 26.0; IBM Corp., Armonk, NY, USA).

\section{Analysis and descriptive statistics of the eye-tracking data}

Data concerning gaze position and pupillary diameter were processed, analyzed, and graphed using custom scripts in MATLAB 2017b (MathWorks). For the $X$ and $Y$ coordinates of gaze position and pupillary diameter, the mean value between the right and left eyes was calculated if measurements were successful in both eyes. If measurements were successful in only one eye, the data from that eye were used.

\section{Eye-tracking analyses of gaze allocation}

To identify differences in gaze allocation between PGs and NPGs, a three-step data analysis process was applied to the coordinates of gaze positions. First, in the decision, feedback, and fixation phases, the ratio of gaze allocation time to total time was calculated for each pixel and participant. Second, the ratios of gaze allocation time for each pixel were compared between PGs and NPGs using two-sample t-tests. 
Third, for the left and right sides in the decision and feedback phases (and for the center in the fixation phase), the midpoints between the peaks of the PGs and NPGs were defined as the foci. Then, in analysis zones bordered by concentric circles centered at the foci and increasing in radius in 10-pixel increments (from 10 to 200 pixels), pixels that showed a significantly greater ratio of gaze allocation time in PGs than in NPGs (PG-dominant pixels) and vice versa (NPG-dominant pixels) were counted. The proportion of significant pixels relative to all pixels was calculated for each zone. This step was completed if nonrandom distributions centered on the foci were observed. Correction for multiple tests was not performed because the probability of false-positives was equal between the PG- and NPG-dominant pixels. In addition, to determine whether the ratio of gaze allocation time toward the left and right pictures differed between PGs and NPGs, the summed ratios of gaze allocation to the left and to the right pictures relative to the total time were analyzed by two-way ANOVA with side (left and right) and group (PGs and NPGs) as independent variables. This analysis was done for the decision, feedback, and fixation phases.

\section{Eye-tracking analyses of pupillary dynamics}

To determine temporal changes in pupillary diameter under each experimental condition, three-step data processing was performed ${ }^{14,15}$. First, invalid samples (e.g., those with blinks) and the three samples before and after the invalid samples were removed to eliminate outliers. Blanks were interpolated using the piecewise cubic Hermite interpolating polynomial method. ${ }^{47}$ Second, the interpolated data were smoothed using a Savitzky-Golay filter with \pm 200 -ms width to reduce sampling noise. ${ }^{48}$ Third, the smoothed data were z-normalized within each individual, and the mean of the time points within each trial was calculated under each of six conditions generated by combining the factors of motivation (reward, punishment, and control) and result (congruent and incongruent). The resulting data were analyzed to compare the influence of reward and punishment between the PGs and NPGs. The differences between the congruent and incongruent conditions at each time point were calculated under the reward, punishment, and control conditions, and each result for the reward and punishment conditions was compared with the results obtained under the control condition using two-sample t-tests.

This analysis made no a priori assumptions regarding the shape of the pupillary response waveform ${ }^{15}$.

\section{Declarations}

\section{Acknowledgments}

This study was supported by JKA through funds from KEIRIN RACE and JSPS KAKENHI (grant numbers $17 K 18116$ and $21 \mathrm{~K} 03148$ for Y.S. and $18 \mathrm{H} 03663$ for A.M.), and by funds from JSPS Topic-Setting Program to Advance Cutting-Edge Humanities and Social Sciences Research Area Cultivation for A. M.

\section{Author contributions}

Y.S. and A.M. conceived of the idea and designed the experiment; Y.S. designed and carried out the experiment, analyzed the data, and wrote the manuscript in consultation with A.M. 


\section{Conflicts of interest}

None of authors has any potential conflicts of interest.

\section{References}

1. American Psychiatric Association. Diagnostic and Statistical Manual of Mental Disorders, Fifth Edition (American Psychiatric Association Publishing, 2013). doi:10.1176/appi.books.9780890425596.

2. Hodgins, D. C., Stea, J. N. \& Grant, J. E. Gambling disorders., 378, 1874-1884 (2011).

3. Blaszczynski, A. \& Silove, D. Pathological gambling: Forensic issues. Aust. N. Z. J. Psychiatry, 30, 358-369 (1996).

4. Grant, J. E. \& Chamberlain, S. R. Gambling disorder and its relationship with substance use disorders: Implications for nosological revisions and treatment. Am. J. Addict, 24, 126-131 (2015).

5. Abbott, M. W. Gambling and gambling-related harm: recent World Health Organization initiatives., 14, https://doi.org/doi:10.1016/j.puhe.2020.04.001. (2020).

6. Zommara, N. M., Takahashi, M., Ounjai, K. \& Lauwereyns, J. A gaze bias with coarse spatial indexing during a gambling task. Cogn. Neurodyn, 12, 171-181 (2018).

7. Simonovic, B., Stupple, E. J. N., Gale, M. \& Sheffield, D. Performance under stress: An eye-tracking investigation of the lowa gambling task (IGT). Front. Behav. Neurosci, 12, 1-10 (2018).

8. Cherkasova, M. V. et al. Win-concurrent sensory cues can promote riskier choice. J. Neurosci, 38, 10362-10370 (2018).

9. Hønsi, A., Mentzoni, R. A., Molde, H. \& Pallesen, S. Attentional Bias in Problem Gambling: A Systematic Review. Journal of Gambling Studies, 29, 359-375 (2013).

10. Eckstein, M. K., Guerra-Carrillo, B., Miller Singley, A. T. \& Bunge, S. A. Beyond eye gaze: What else can eyetracking reveal about cognition and cognitive development? Dev. Cogn. Neurosci, 25, 69-91 (2017).

11. Ebitz, B. R. \& Moore, T. Both a gauge and a filter: Cognitive modulations of pupil size. Front. Neurol, 10, 1-14 (2019).

12. Schneider, M. et al. Pupil dilation during reward anticipation is correlated to depressive symptom load in patients with major depressive disorder. Brain Sci, 10, 1-15 (2020).

13. Schneider, M., Leuchs, L., Czisch, M. \& Sämann, P. G. Spoormaker, V. I. Disentangling reward anticipation with simultaneous pupillometry / fMRI., 178, 11-22 (2018).

14. Watanabe, N., Bhanji, J. P., Ohira, H. \& Delgado, M. R. Reward-Driven Arousal Impacts Preparation to Perform a Task via Amygdala-Caudate Mechanisms. Cereb. Cortex, 29, 3010-3022 (2019).

15. Satterthwaite, T. D. et al. Dissociable but inter-related systems of cognitive control and reward during decision making: Evidence from pupillometry and event-related fMRI., 37, 1017-1031 (2007). 
16. Lavín, C., Martín, R. S. \& Jubal, E. R. Pupil dilation signals uncertainty and surprise in a learning gambling task. Frontiers in Behavioral Neuroscience, 7, 218 (2013).

17. Preuschoff, K., 't Hart, B. M. \& Einhäuser, W. Pupil dilation signals surprise: Evidence for noradrenaline's role in decision making. Front. Neurosci, 5, 1-12 (2011).

18. Lesieur, H. R. \& Blume, S. B. The South Oaks Gambling Screen (SOGS): A new instrument for the identification of Pathological gamblers. Am. J. Psychiatry, 144, 1184-1188 (1987).

19. Jacobs, J. Experiments on “Prehension”. Mind, 12, 75-79 (1887).

20. Rey, A. L'examen psychologique dans les cas d'encéphalopathie traumatique. (Les problems.) [The psychological examination in cases of traumatic encepholopathy. Problems]. Arch. Psychol. (Geneve), 28, 286-340 (1941).

21. Osterrieth, P. Le test de copie d'une figure complexe; contribution à l'étude de la perception et de la mémoire [Test of copying a complex figure; contribution to the study of perception and memory]. Arch. Psychol. (Geneve), 30, 206-356 (1944).

22. Carver, C. S., White, T. L. \& Behavioral Inhibition Behavioral Activation, and Affective Responses to Impending Reward and Punishment: The BIS/BAS Scales. J. Pers. Soc. Psychol, 67, 319-333 (1994).

23. Torrubia, R., Ávila, C., Moltó, J. \& Caseras, X. The Sensitivity to Punishment and Sensitivity to Reward Questionnaire (SPSRQ) as a measure of Gray's anxiety and impulsivity dimensions. Pers. Individ. Dif, 31, 837-862 (2001).

24. Zuckerman, M. Sensation seeking: beyond the optimal level of arousal (Lawrence Erlbaum Associates, 1979).

25. Furusawa, T. An attempt to construct the sensation seeking scale-abstract expression. Japanese J. Psychol, 60, 180-184 (1989).

26. McGrath, D. S., Meitner, A. \& Sears, C. R. The specificity of attentional biases by type of gambling: An eye-tracking study. PLoS One, 13, 1-16 (2018).

27. Zack, M. \& Poulos, C. X. Amphetamine Primes Motivation to Gamble and Gambling-Related Semantic Networks in Problem Gamblers. Neuropsychopharmacology, 29, 195-207 (2004).

28. McCusker, C. G. \& Gettings, B. Automaticity of cognitive biases in addictive behaviours: Further evidence with gamblers. Br. J. Clin. Psychol, 36, 543-554 (1997).

29. Brevers, D. et al. Time Course of Attentional Bias for Gambling Information in Problem Gambling. Psychol. Addict. Behav, 25, 675-682 (2011).

30. Gammeri, R., lacono, C., Ricci, R. \& Salatino, A. Unilateral spatial neglect after stroke: Current insights. Neuropsychiatr. Dis. Treat, 16, 131-152 (2020).

31. Heilman, K. M., Valenstein, E. \& Watson, R. T. Neglect and related disorders. Semin. Neurol, 20, 463470 (2000).

32. Davidson, R. Anterior Cerebral Asymmetry and the Nature of Emotion. Brain Cogn, 151, 125-151 (1992). 
33. Davidson, R. J. What does the prefrontal cortex 'do' in affect: Perspectives on frontal EEG asymmetry research. Biol. Psychol, 67, 219-234 (2004).

34. Harmon-Jones, E. \& Gable, P. A. On the role of asymmetric frontal cortical activity in approach and withdrawal motivation: An updated review of the evidence., 55, e12879 (2018).

35. De Pascalis, V., Cozzuto, G., Caprara, G. V. \& Alessandri, G. Relations among EEG-alpha asymmetry, BIS/BAS, and dispositional optimism. Biol. Psychol, 94, 198-209 (2013).

36. Balconi, M. \& Mazza, G. Lateralisation effect in comprehension of emotional facial expression: A comparison between EEG alpha band power and behavioural inhibition (BIS) and activation (BAS) systems. Laterality, 15, 361-384 (2010).

37. Wacker, J., Mueller, E. M., Pizzagalli, D. A., Hennig, J. \& Stemmler, G. Dopamine-D2-Receptor Blockade Reverses the Association Between Trait Approach Motivation and Frontal Asymmetry in an Approach-Motivation Context. Psychol. Sci, 24, 489-497 (2013).

38. Balconi, M., Finocchiaro, R. \& Canavesio, Y. Reward-system effect (BAS rating), left hemispheric 'unbalance' (alpha band oscillations) and decisional impairments in drug addiction. Addict. Behav, 39, 1026-1032 (2014).

39. Balconi, M., Finocchiaro, R. \& Canavesio, Y. Left hemispheric imbalance and reward mechanisms affect gambling behavior: The contribution of the metacognition and cortical brain oscillations. Clin. EEG Neurosci, 46, 197-207 (2015).

40. Tomer, R. Attentional bias as trait: Correlations with novelty seeking., 46, 2064-2070 (2008).

41. Brevers, D., He, Q., Xue, G. \& Bechara, A. Neural correlates of the impact of prior outcomes on subsequent monetary decision-making in frequent poker players. Biol. Psychol, 124, 30-38 (2017).

42. Campbell-Meiklejohn, D. K., Woolrich, M. W., Passingham, R. E. \& Rogers, R. D. Knowing When to Stop: The Brain Mechanisms of Chasing Losses. Biol. Psychiatry, 63, 293-300 (2008).

43. Gainsbury, S. M., Suhonen, N. \& Saastamoinen, J. Chasing losses in online poker and casino games: Characteristics and game play of Internet gamblers at risk of disordered gambling. Psychiatry Res, 217, 220-225 (2014).

44. Shaffer, H. J., LaBrie, R., Scanlan, K. M. \& Cummings, T. N. Pathological gambling among adolescents: Massachusetts Gambling Screen (MAGS). J. Gambl. Stud, 10, 339-362 (1994).

45. Erdfelder, E., FAul, F., Buchner, A. \& Lang, A. G. Statistical power analyses using G*Power 3.1: Tests for correlation and regression analyses. Behav. Res. Methods, 41, 1149-1160 (2009).

46. Abe, N. et al. Encoding- and retrieval-related brain activity underlying false recognition. Neurosci. Res, 76, 240-250 (2013).

47. Fritscht, F. N. \& Carlson, R. E. Monotone piecewise cubic interpolation. SIAM J. Numer. Anal, 17, 238246 (1980).

48. Orfanidis, S. J. Introduction to signal processing (Prentice Hall, 1996).

\section{Figures}




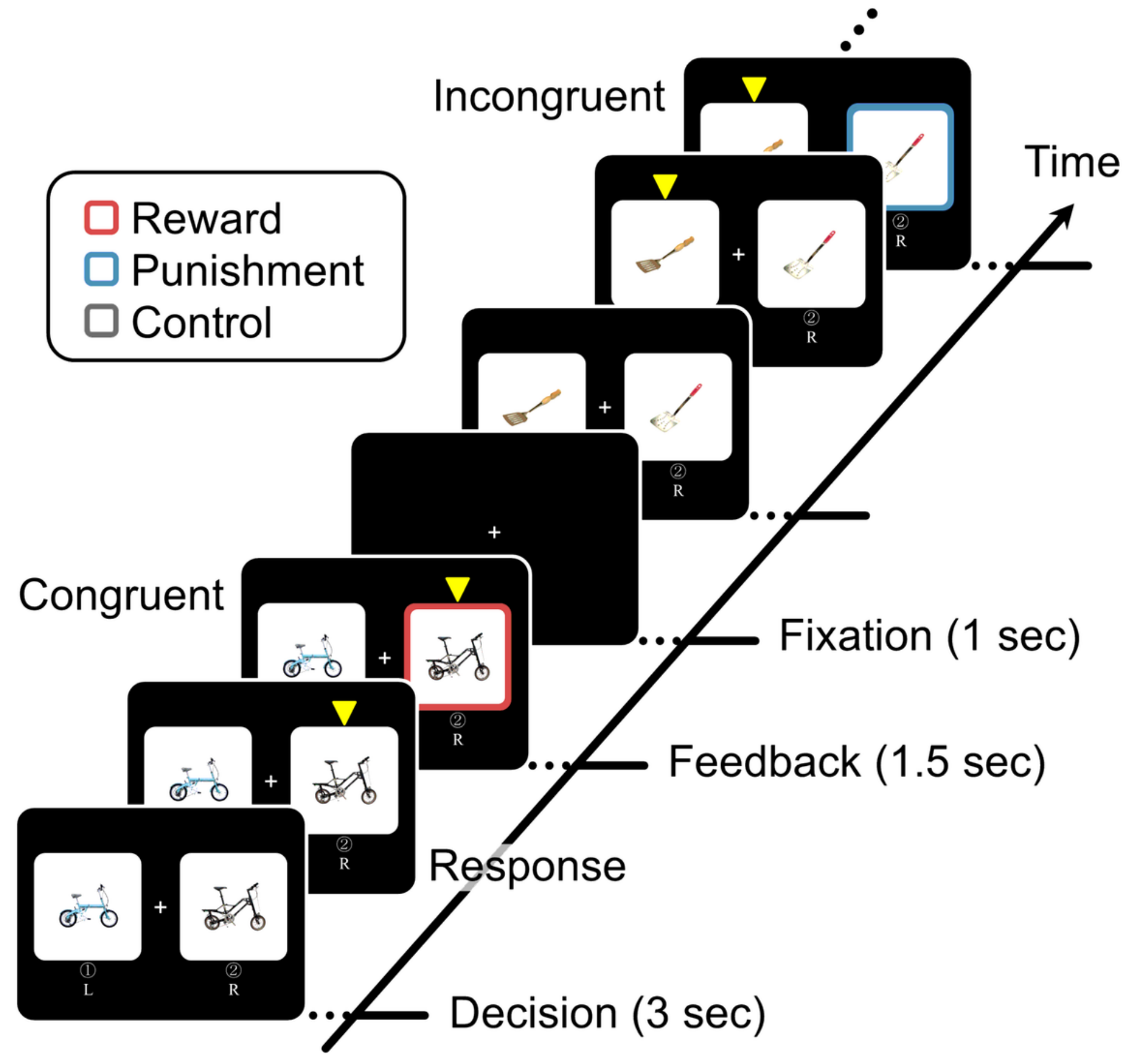

Figure 1

Experimental design. Participants were required to choose the right- or left-hand picture during the decision phase. An inverted triangle was presented above the chosen picture. In the feedback phase, if the chosen picture was framed by a red line, the participant received a monetary reward. If the chosen picture was framed by a blue line, the participant received a monetary punishment. If the chosen picture was framed by a gray line or was not framed, the participant received no reward or punishment. 
(a)
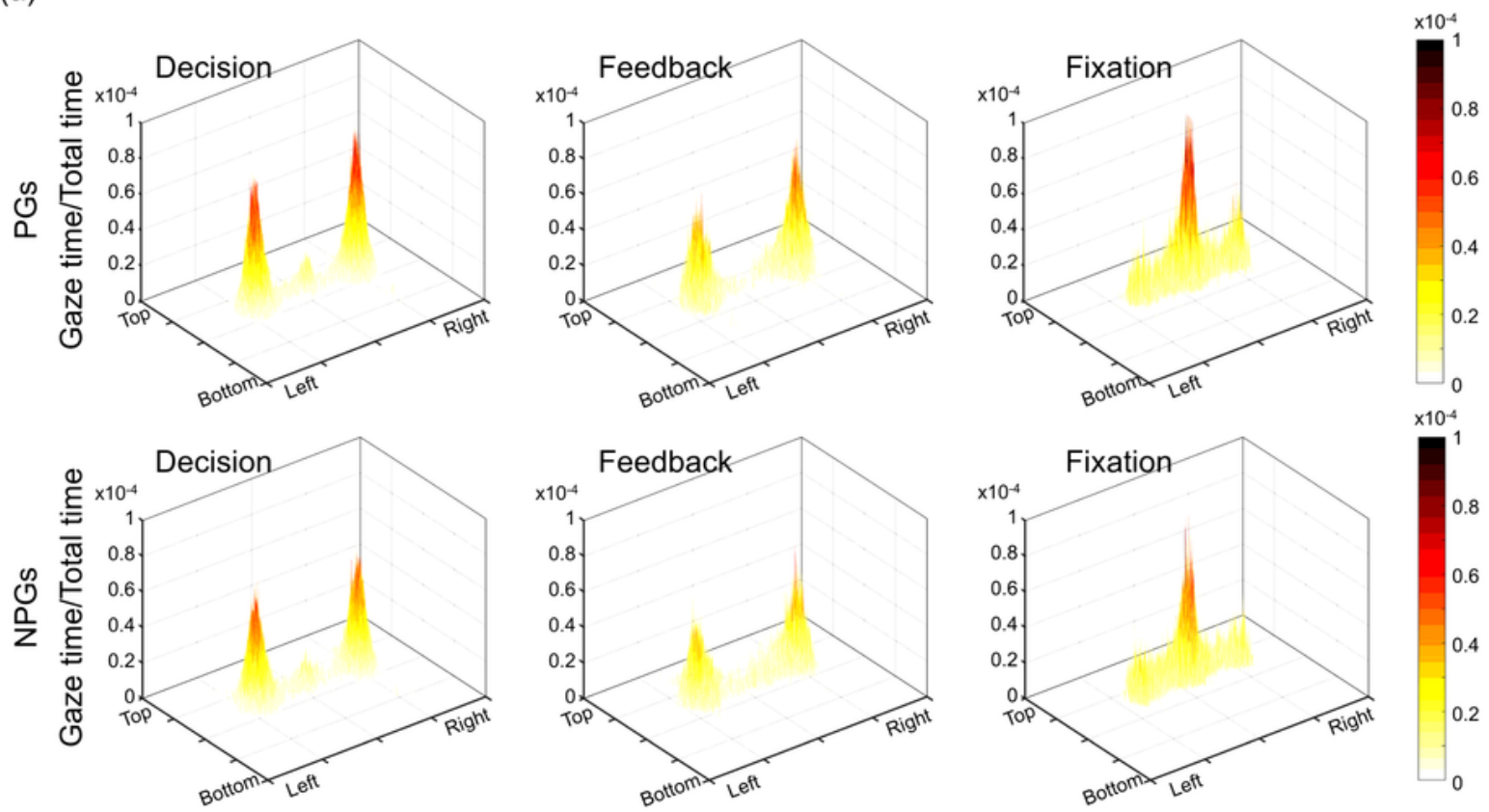

(b)
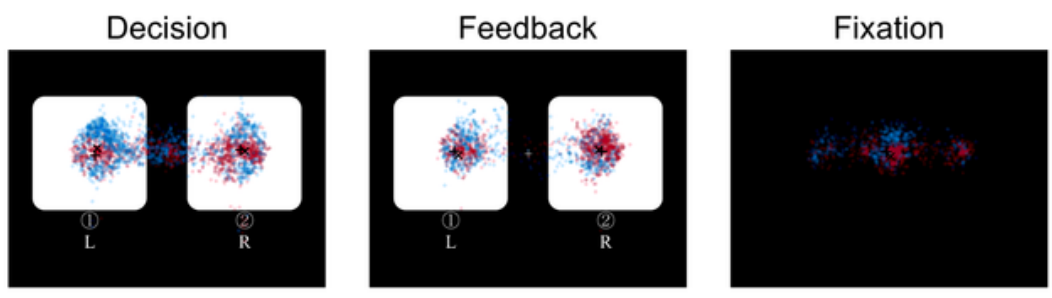

Significant pixel

PG-dominant Peak

(PGs>NPGs) x PGs

NPG-dominant + NPGs

(c)
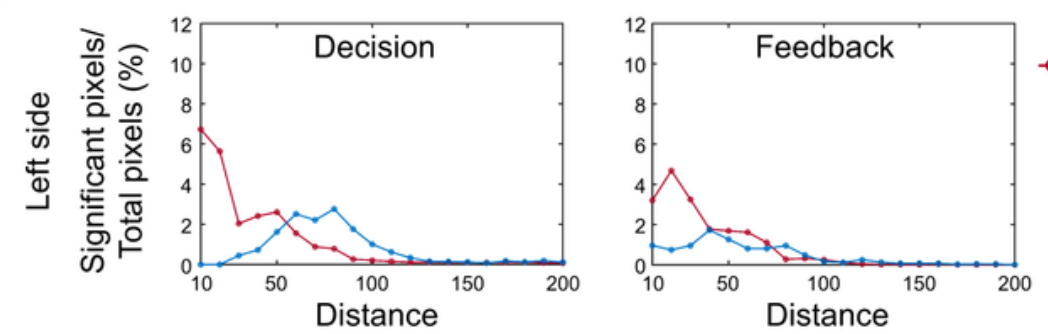

PG-dominant
$($ PGs $>$ NPGs) $\underset{(\text { NPG-dominant }}{(\text { NPGs }>\text { PGs) }}$
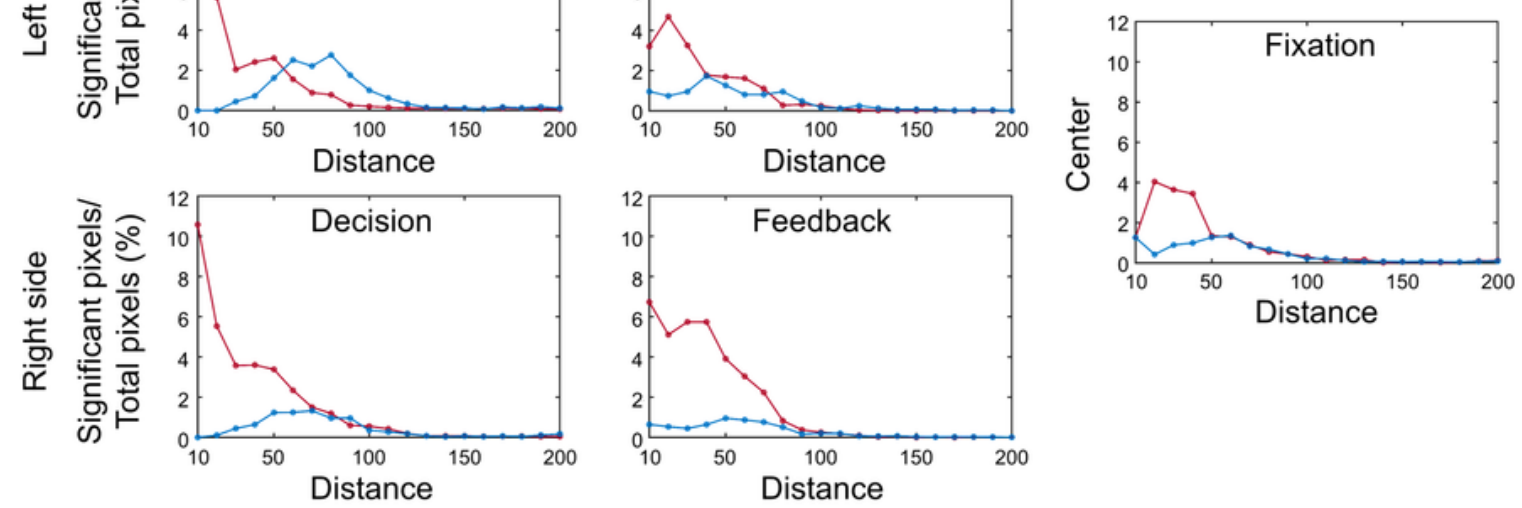

\section{Figure 2}

Gaze allocation. (a) Ratio of gaze allocation time for each pixel. The bottom indicated by the $x$ - and $y$ axes represents the screen, and the z-axis represents the ratio. (b) Differences between PGs and NPGs. Red dots represent pixels in which the ratio of gaze allocation time was significantly greater in PGs than in NPGs (PG-dominant). Blue dots represent pixels in which the ratio of gaze allocation time was significantly greater in NPGs than in PGs (NPG-dominant). (c) Distributions of significant differences at 
each distance (increasing in 10-pixel increments) from the midpoints between the peaks of the PGs and NPGs. The red line represents the proportion of PG-dominant pixels. The blue line represents the proportion of NPG-dominant pixels.
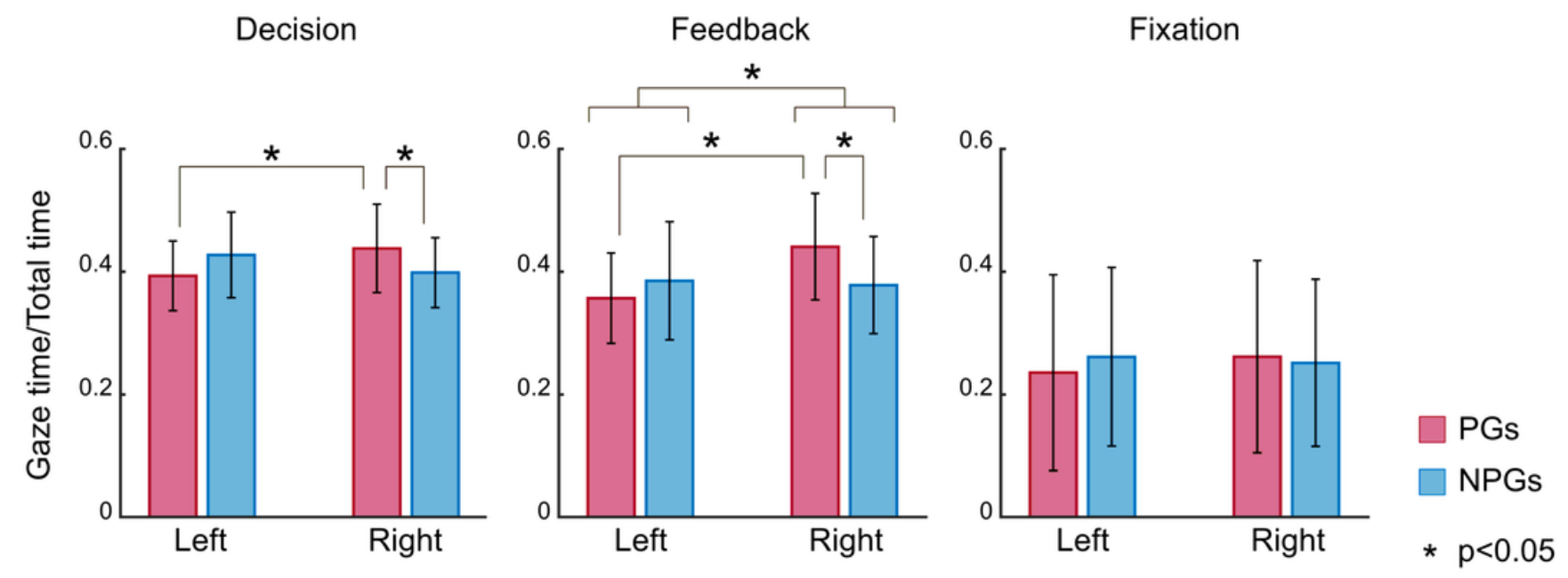

\section{Figure 3}

Ratio of gaze allocation time for zones corresponding to the left and right pictures. Error bars represent SDs. ${ }^{\star} p<0.05$ 
(a)

PGs

NPGs

Congruent
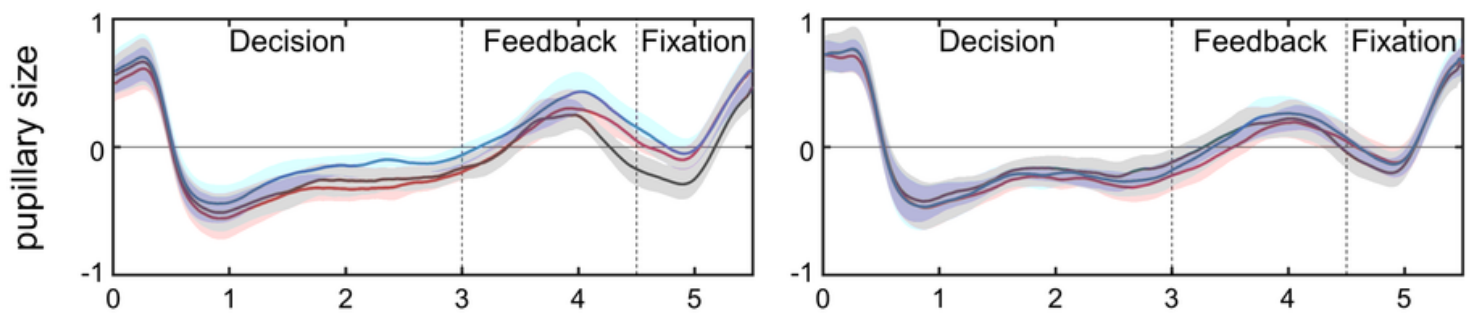

Incongruent
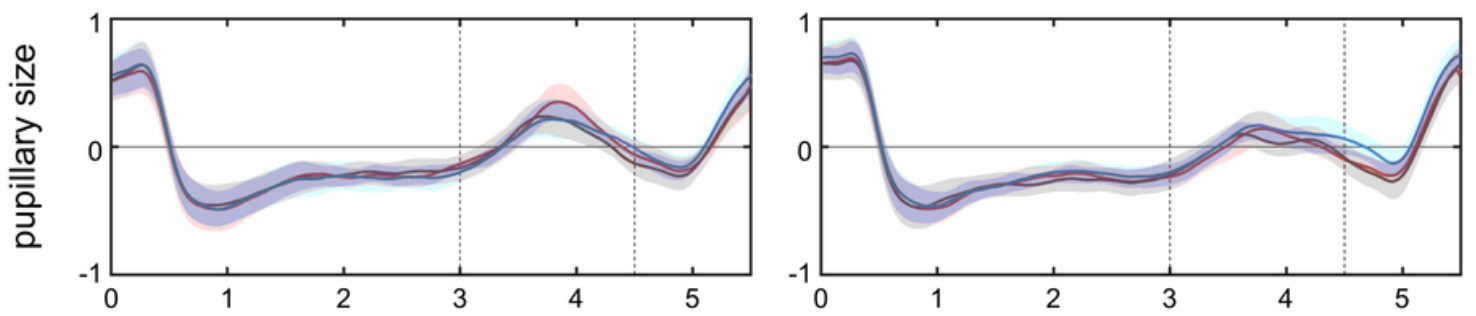

(b)
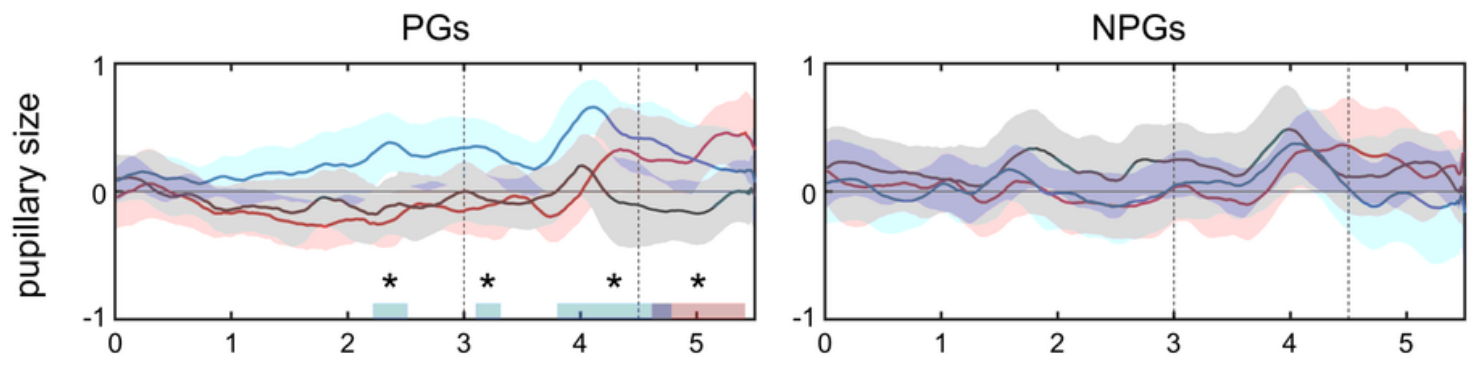

- Reward

- Punishment

- Control

* $p<0.05$

\section{Figure 4}

Pupillary dynamics. Dashed lines represent the onset of the feedback and fixation phases. Colored bands along the lines represent $95 \%$ confidence intervals. (a) Pupillary dynamics in each experimental condition. (b) Differences between the congruent and incongruent conditions in the reward, punishment, and control conditions. Boxes with asterisks along the $\mathrm{x}$-axis represent significant differences. Red boxes represent significant differences between the reward and control conditions. Blue boxes represent significant differences between the punishment and control conditions. ${ }^{*} p<0.05$ 